\title{
Speeding up quantum dissipative dynamics of open systems with kernel methods
}

\author{
Arif Ullah* and Pavlo O. Dral* \\ State Key Laboratory of Physical Chemistry of Solid Surfaces, Fujian Provincial Key \\ Laboratory of Theoretical and Computational Chemistry, Department of Chemistry, and \\ College of Chemistry and Chemical Engineering, Xiamen University, Xiamen 361005, \\ China \\ E-mail: ua2024@xmu.edu.cn; dral@xmu.edu.cn
}

\begin{abstract}
The future forecasting ability of machine learning (ML) makes ML a promising tool for predicting long-time quantum dissipative dynamics of open systems. In this Article, we employ nonparametric machine learning algorithm (kernel ridge regression as a representative of the kernel methods) to study the quantum dissipative dynamics of the widely-used spin-boson model. Our ML model takes short-time dynamics as an input and is used for fast propagation of the long-time dynamics, greatly reducing the computational effort in comparison with the traditional approaches. Presented results show that the ML model performs well in both symmetric and asymmetric spin-boson models. Our approach is not limited to spin-boson model and can be extended to complex systems.
\end{abstract}




\section{Introduction}

With the realization that isolated systems do not exist in the real world, we have to deal with open quantum systems, where we need to consider that the system and the surrounding environment can exchange energy, particles and/or quantum phase. Spin-boson (SB) model is one of the well-known models which is widely used to understand the effects of the surrounding environment on the system. SB model describes a two-state system coupled to infinite number of non-interacting harmonic oscillators. Understanding quantum dissipative dynamics of SB model (or in general open quantum system) has applications in wide range of settings such as quantum computing, ${ }^{1]}$ quantum memories, ${ }^{[2}$ quantum electrodynamics, organic solar cells, ${ }^{[5]}$ superconducting junctions, ${ }^{[6]}$ quantum biology, ${ }^{[7}$ quantum optics,,$\left[\frac{8]}{\text { quan- }}\right.$ tum transport, $\frac{910}{10}$ defect tunneling in solids, $\frac{1112}{12}$ quantum dots, 13114 and colour centres and Cooper pair boxes. ${ }^{[15[16}$ In addition, SB model has remained a key-model for testing and comparison of open quantum system theories before extending them to complex systems. A large number of numerical methods has been developed to study SB model such as numerical renormalization group (NRG) method, $\frac{17 / 18}{1}$ the density matrix renormalization group (DMRG) method, $\frac{1920}{2}$ the quantum Monte Carlo (QMC) method, 2122 the time evolving density matrix using orthogonal polynomial algorithm (TEDOPA), 2324 the reaction coordinate mapping, ${ }^{[25}$ the multi-configuration time-dependent Hartree (MCTDH) method ${ }^{[26[27]}$ and its extensions $^{28}$ the Nakajima-Zwanzig generalized quantum master equation(GQME), 29130 transfer tensor method (TTM) ${ }^{31+33}$ and numerical variational method(NVM). ${ }^{34}$ Regardless of their success, these methods have there own limitations in applicability and accuracy.

In addition to these methods, another popular approach is classical mapping-based approach (Meyer-Miller-Stock-Thoss mapping, ${ }^{\sqrt[35+59]{39}}$ spin-based mapping ${ }^{\sqrt[40141]{61}}$ and so on), where system and surrounding environment dynamics is described by classical trajectories. Classical mapping-based approaches are more feasible but because of the classical description, they fail to properly describe the dynamics of the system especially at low temperature. Coming back to exact quantum methods, Makri and coauthors proposed quasiadiabatic propagator 
path integral (QUAPI) scheme. $\stackrel{42143}{ }$ In QUAPI approach, all correlation effects are included over a finite time $\tau=K \delta t$ and correlation effects beyond $\tau$ are neglected. Good convergence requires time $\tau$ to be as large as possible and the Trotter increment $\delta t$ as small as possible. In QUAPI approach, the growing computational cost with the increase of system size and time $\tau$ limits its applicability for large complex systems.

An alternative numerical exact bench-marking approach is the hierarchical equations of motion $(\mathrm{HEOM})^{44} \sqrt[53]{5}$ approach pioneered by Tanimura and Kubo. ${ }^{54}$ HEOM approach captures the combined effects of system-environment dissipation, non-Markovian memory effect, and many-body correlation in a non-perturbative manner. In this approach, a hierarchy of deterministic differential equations are constructed by using a set of memory basis functions to unravel the correlation function of the environment. The size of HEOM depends on two factors; the number of memory basis functions $M$ and the depth of the hierarchy $L$, which depends critically on the strength of the system-environment interaction and many-body correlation. In the case of low temperature and strong dissipative interaction, good convergence requires large $M$ and $L$, which inevitably makes HEOM approach rather expensive. Such a drawback has restrained the use of HEOM method in the ultra-low temperature regime.

Another approach with no temperature limitation, is the trajectory based stochastic equation of motion (SEOM) approach. In stochastic formulation, the influence of environment on the system is captured by stochastic auxiliary fields. The SEOM for boson environment has been established and adopted by many authors. ${ }^{55}[68$ Recently, the stochastic approach has been extended to open quantum system with fermion environment. $\frac{69}{71}$ Numerous amount of research work can be found on the applications of SEOM. ${ }^{775}$ SEOM approach can be used in both low- and high-temperature cases, although at low temperature, a good convergence needs more trajectories. The bottleneck of SEOM approach for complex systems is the increase in the number of noises with the number of states, which as a result makes SEOM approach hard to converge in the long-time limit. 
In the past decade, machine learning (ML) has found its applications in all fields of science. In the field of chemical physics, ML has seen many applications, $\frac{76 / 77}{}$ such as to con-

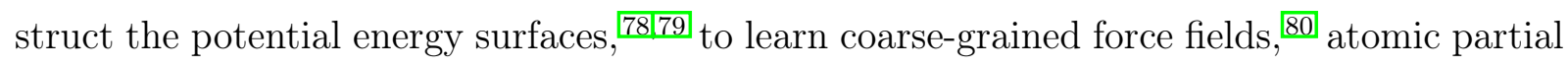
charges,, 81 dielectric constants in crystals,,$\frac{82}{86}$ absorption cross sections,,$\frac{831}{10}$ and excited state dynamics. $.84 \sqrt[86]{8 L}$ approaches (recurrent neural network and non-linear autoregressive neural networks) have been employed to simulate the quantum dissipative dynamics of SB and Landau-Zener models. $\stackrel{8788}{ }$ Recently Rodríguez and Kananenka ${ }^{89}$ have used the parametric ML model based on convolutional neural networks to study the excitation energy transfer in a dimer, where they suggested to use short-time dynamics as input for ML. In their approach, ML was trained on reduced density matrix elements and required computationally expensive fitting of ca. 3 million parameters on ca. 4 thousand trajectories.

Realizing the power of nonparametric machine learning algorithms, in this paper, we are utilizing the kernel ridge regression (KRR) model to simulate the quantum dissipative dynamics of very general SB model, which is a typical model for benchmarking different approaches. Nonparametric models based on kernel methods, to which KRR belongs, often provide more accurate ML models as was shown in several independent studies. ${ }^{90}\left[\frac{92}{\mathrm{In}}\right.$ addition, the problem of fitting their parameters has closed solution as it is the convex optimization problem, making them attractive for training ML models. In our approach we train the ML model directly on the expectation value of $\hat{\sigma}_{z}$ (aka the population difference), making our approach more robust avoiding unphysical behavior, which may potentially arise while training on the reduced density matrix elements. After training, our KRR model takes rather short-time quantum dynamics as input and predicts the long-time dynamics as an output making our model a promising cost-effective tool for open quantum systems. Interestingly, our approach only required 450 training trajectories and fitting of 72 thousand parameters. It accurately predicts the long-time dynamics in all cases: from weak systemenvironment coupling to strong system-environment coupling and from weak environment to strong environment cases. In our study, we included both the symmetric and asymmetric 
cases of the SB model.

The rest of the paper is organized as follows. In the Theory section, we review the theory of the SB model and present our KRR approach. Next section is Results and Discussion which is followed by Concluding Remarks.

\section{Theory}

\section{Spin-boson model}

The theory behind the SB model is well-explored by many authors, ${ }^{21 / 35144 \mid 45155156]}$ but for the sake of completeness, we summarize it here too. The well-known SB model describes a two-level system coupled with the outside environment. The environment consists of infinite number of non-interacting harmonic oscillators. The total Hamiltonian is written as $(\hbar=1)$

$$
\begin{aligned}
& \hat{H}=\hat{H}_{\mathrm{s}}+\hat{H}_{\mathrm{env}}+\hat{H}_{\mathrm{s}-\mathrm{env}}, \\
& \hat{H}=\frac{1}{2} \epsilon \hat{\sigma}_{z}+\frac{1}{2} \Delta \hat{\sigma}_{x}+\sum_{k} \omega_{k} \hat{b}_{k}^{\dagger} \hat{b}_{k}+\frac{1}{2} \hat{\sigma}_{z} \hat{F},
\end{aligned}
$$

where $\hat{H}_{\mathrm{s}}, \hat{H}_{\mathrm{env}}$ and $\hat{H}_{\mathrm{s}-\mathrm{env}}$ represent Hamiltonians of the 2-level system, environment and their interaction, respectively. $\hat{\sigma}_{x}$ and $\hat{\sigma}_{z}$ are Pauli matrices and $\epsilon$ is the energy bias of the two states, i.e., $|e\rangle$ and $|g\rangle . \Delta$ is the tunneling matrix element of the two states. $b_{k}^{\dagger}$ is the creation operator of the environment mode $k$. The environment mode $k$ interacts with the system via operator $\hat{F}_{k}=\frac{c_{k}}{\sqrt{2 \omega_{k}}}\left(\hat{b}_{k}+\hat{b}_{k}^{\dagger}\right)$, where $c_{k}$ is the coupling strength. The total interaction operator $F$ can be written as $\hat{F}=\sum_{k} \frac{c_{k}}{\sqrt{2 \omega_{k}}}\left(\hat{b}_{k}+\hat{b}_{k}^{\dagger}\right)$.

It is common to consider that at initial time $t=0$, the interaction between system and the surrounding environment is zero and the environment is in thermal equilibrium state. The influence of the environment can be described by a two-time correlation function of operator $\hat{F}$, i.e., $C(t)=\langle\hat{F}(t) \hat{F}(0)\rangle$. In the case of no interaction with the system, $C(t)$ can 
be written as

$$
C(t)=\frac{1}{\pi} \int_{0}^{\infty} \mathrm{d} \omega J(\omega)\left(\operatorname{coth}\left(\frac{\omega \beta}{2}\right) \cos (\omega t)-\mathrm{i} \sin (\omega t)\right)
$$

where $J(\omega)$ is the spectral density

$$
J(\omega)=\frac{\pi}{2} \sum_{k} \frac{c_{k}^{2}}{\omega_{k}} \delta\left(\omega-\omega_{k}\right) .
$$

As we are only interested in the dynamics of the system, we can trace out the environment degrees of freedom,

$$
\hat{\rho}_{\mathrm{s}}(t)=\operatorname{Tr}_{\mathrm{env}}\left[\hat{U}(t, 0) \hat{\rho}_{\mathrm{T}}(0) \hat{U}^{\dagger}(t, 0)\right]
$$

here $\hat{\rho}_{\mathrm{s}}(t)$ is the reduced density matrix of the system at time $t$ while $\hat{\rho}(0)$ is the initial density matrix of the composite system governed by $\hat{H} \cdot \hat{U}(t, 0)$ and $\hat{U}^{\dagger}(t, 0)$ are operators for forward and backward propagation in time, respectively. Different approaches deal with Eq.(4) differently as mentioned in Introduction section. For more details on a specific approach, readers are referred to the corresponding references.

\section{Kernel ridge regression}

In kernel ridge regression (KRR), which is also known as Kernel Regularized Least Squares, $\underline{93}$ the approximating function $f(\mathbf{x})$ for a vector of input values $\mathbf{x}$ is defined as

$$
f(\mathbf{x})=\sum_{i=1}^{N} \alpha_{i} k_{i}\left(\mathbf{x}, \mathbf{x}_{i}\right)
$$

here $N$ is the number of training points and $\boldsymbol{\alpha}$ are the regression coefficients. The kernel function $k\left(\mathbf{x}, \mathbf{x}_{i}\right)$ takes two vectors $\boldsymbol{x}$ and $\boldsymbol{x}_{i}$ from the input space and measures the distance between them. 
In this work we use the Gaussian kernel as implemented in the MLatom package: 96 98

$$
k\left(\mathbf{x}, \mathbf{x}_{i}\right)=\exp \left(-\frac{\left\|\mathbf{x}-\mathbf{x}_{i}\right\|_{2}^{2}}{2 \sigma^{2}}\right)
$$

as our tests showed that it has a good performance for our application and other kernels such as those from a family of popular Matérn kernels are not better. In the Gaussian kernel, we have only one hyperparameter $\sigma$, defining the length scale. Intuitively, the Gaussian kernel measures similarity between the vectors $\mathbf{x}$ and $\mathbf{x}_{i}$. The output of these kernels increases as $\left\|\mathbf{x}-\mathbf{x}_{i}\right\| \rightarrow 0$ and becomes unity at $\mathbf{x}=\mathbf{x}_{i}$ while for large distance $\left\|\mathbf{x}-\mathbf{x}_{i}\right\| \rightarrow \infty$, the kernel $k\left(\mathbf{x}, \mathbf{x}_{i}\right)$ tends to zero. After choosing the kernel function, we need to find the regression coefficients $\boldsymbol{\alpha}$ in Eq. (5). It is done by minimization a squared error loss function $\frac{7695196}{}$

$$
\left.\min _{\alpha} \sum_{i}^{N}\left(f\left(x_{i}\right)-y_{i}\right)\right)^{2}+\lambda \boldsymbol{\alpha}^{T} \mathbf{K} \boldsymbol{\alpha}
$$

here $\mathbf{y}$ is the target output vector, $\mathbf{K}$ is the kernel matrix and $\lambda$ denotes a non-negative regularization hyperparameter. In above equation, the second term is usually added to stop KRR model from giving too much weight to a single point. ${ }^{99}$ After simple algebra, Eq.(7) leads to

$$
\boldsymbol{\alpha}=(\mathbf{K}+\lambda \mathbf{I})^{-1} \mathbf{y},
$$

here $\mathbf{I}$ is the identity matrix.

\section{Data and Training}

We consider that our system is initially in the excited state (i.e., $|e\rangle)$. For the environment, the Drude-Lorentz spectral density is considered

$$
J(\omega)=2 \lambda \frac{\omega \omega_{\mathrm{c}}}{\omega^{2}+\omega_{\mathrm{c}}^{2}}
$$


where $\omega_{c}$ and $\lambda$ denote the characteristic frequency and the system-environment coupling strength, respectively. In this work, all considered parameters are in atomic units (a.u.). The time-step for integration is $\Delta t=0.05$ and the time of propagation $t$ is fixed to be 20. We generate trajectories of reduced density matrices for all combinations of the following parameters: $\Delta=1, \epsilon \in\{0,1\}, \lambda \in\{0.1,0.2,0.3,0.4,0.5,0.6,0.7,0.8,0.9,1.0\}$, $\omega_{c} \in\{1,2,3,4,5,6,7,8,9,10\}$, and $\beta \in\{0.1,0.25,0.5,0.75,1\}$, here $\beta=1 / k_{\mathrm{B}} T$ with $k_{\mathrm{B}}$ as Boltzmann constant and $T$ as temperature. It should be noted that we include data for both symmetric and asymmetric SB models, i.e., $\epsilon \in\{0,1\}$. With the given set of parameters, 1000 trajectories (500 for symmetric and 500 for asymmetric case) of reduced density matrix are generated with the HEOM approach as implemented in the publicly available QuTiP package. $\frac{100}{}$ As the HEOM method is computationally very expensive at low temperature, we did not include data for very low temperatures.

We train our model directly on the data extracted for the expectation value of $\hat{\sigma}_{z}$ as it is the quantity of interest in the case of spin-boson model. The ML model can be trained for all the elements of the reduced density matrix as done in Ref. 89, but this approach is expensive and may potentially lead to unphysical errors in the expectation value of $\hat{\sigma}_{z}$. Coming back to training our KRR model, the initial training data are raw trajectories, which can be viewed as unsupervised data. To transform it into supervised data, we take a small cut with a time-length $t=t_{m}$ (memory time using terminology of Rodríguez and Kananenka ${ }^{89}$ ) as input trajectory and define the next time step as output. In our case, we considered $t_{m}=4$. Taking the small cut with $t=t_{m}$ as a base trajectory, $N=\left(t-t_{m}\right) / \Delta t_{\text {train }}$ number of supervised trajectories are generated. For training, the $\Delta t_{\text {train }}$ can be larger than the propagation-time step $\Delta t$. In our case, we considered $\Delta t_{\text {train }}=0.1$. Following this procedure, we transformed one unsupervised trajectory with time-length $t$ into $N$ supervised trajectories each with time-length $t_{m}$.

From the data set of 1000 trajectories, 100 randomly chosen trajectories are taken as the hold-out set, which we use to produce results presented in this paper. With $\Delta t_{\text {train }}=0.1$ 
and $t_{m}=4$, the remaining set of unsupervised trajectories of time-length $t=20$ are transformed into 144000 supervised trajectories of time-length $t_{m}=4$. The data set of supervised trajectories is randomly partitioned into two subsets: a training set, which contains $80 \%$ of the data and a validation set with $20 \%$ random trajectories of the set for the optimization of hyperparameters $\sigma$ and $\lambda$ on the logarithmic grid. ${ }^{96}$ For ML calculations we used the MLatom package. ${ }^{96}$ We train two separate models for symmetric and asymmetric cases and the errors for test set are shown in Table 1. Fig. 1 systematically explains all the steps adopted during training and data preparation.

Table 1: Mean absolute error (MAE), Mean square error (MSE) and Root mean square error (RMSE) for the test set.

\begin{tabular}{|l||l|l|l|}
\hline SB models & MAE & MSE & RMSE \\
\hline Symmetric & $9.20 \times 10^{-4}$ & $6.12 \times 10^{-6}$ & $2.47 \times 10^{-3}$ \\
Asymmetric & $1.97 \times 10^{-3}$ & $8.00 \times 10^{-5}$ & $8.94 \times 10^{-3}$ \\
\hline
\end{tabular}




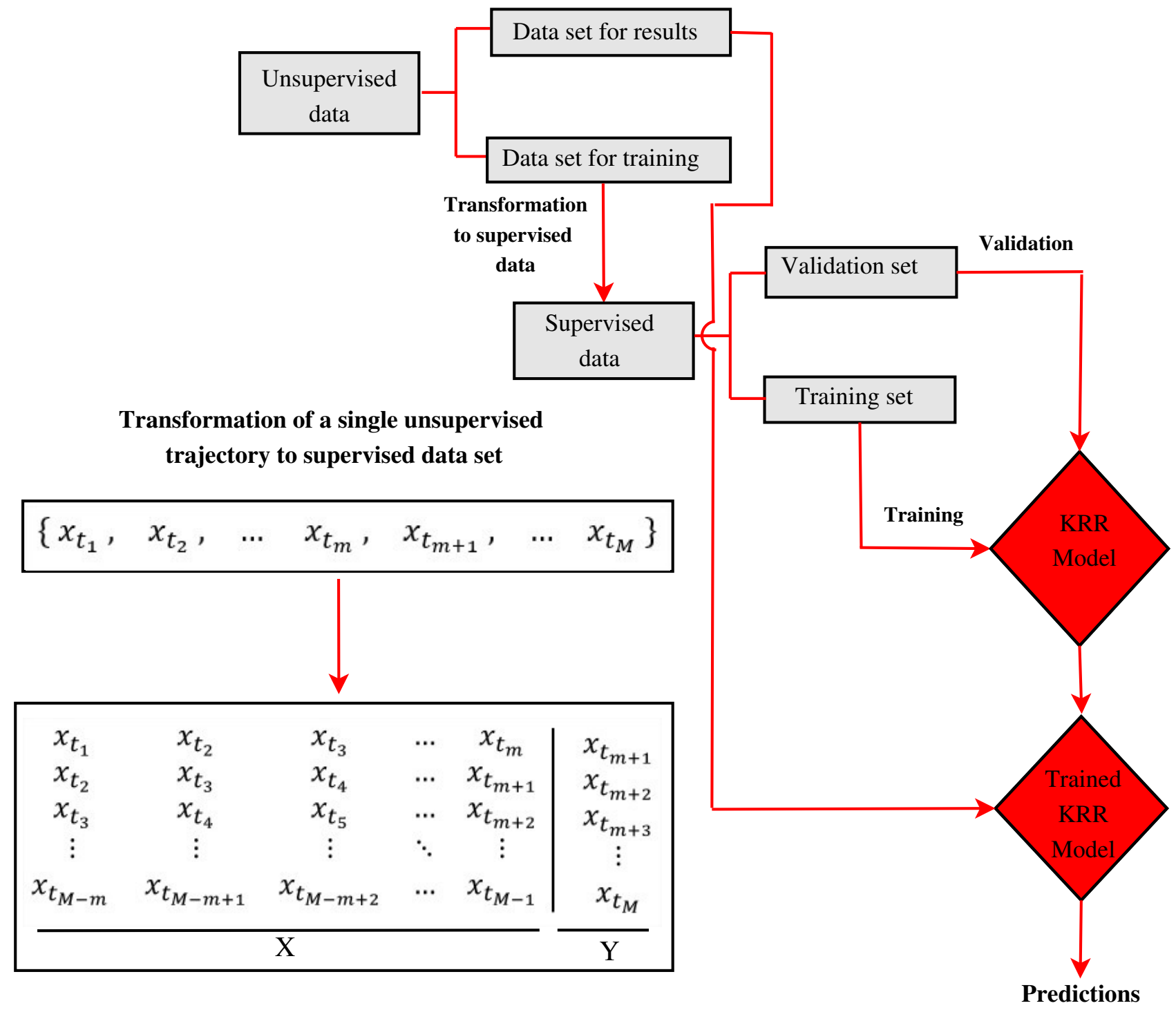

Figure 1: Flowchart of all steps during data preparation, training and predictions. 


\section{Results and Discussion}

After training our KRR model, it takes a short-time trajectory with the time-length $t_{m}$ as input and predicts the value of $\left\langle\hat{\sigma}_{z}\right\rangle$ at the next time step $t_{m+1}$. This gives us the valuable information about the temporal evolution of the state population in contrast to alternative approaches, where ML was used to completely bypass HEOM dynamics and predict the final properties of interest such as transfer times and transfer efficiencies in the pigment-protein complexes. ${ }^{[101}$ We prepare a new input by including the predicted time-step and this process continues until the last time step. It is worth emphasising that the fed input trajectories are unseen to our trained KRR models. Fig. 2 shows results for symmetric SB model with a wide range of parameters. Similarly, Fig. 3 shows results for asymmetric SB model. Fig. 2 and Fig. 3 show small fraction of results. For the remaining results of hold-out test set, interested readers are referred to Supporting Information. The predicted results cover a wide range of scenarios from strong environment to weak environment and from strong coupling to weak coupling.

From Fig. 2 we can see that the predictions by KRR model are very accurate for symmetric cases. For asymmetric SB model, the predicted result given in Fig. S3-(3f) of Supporting Information slightly deviate from the the exact HEOM results in the long-time regime. The possible reason is that the memory-time or in other words, the window-size of the base trajectory $\left(\left|0-t_{m}\right|\right)$ is not enough for this case. In this work, we have considered the same memory time for both symmetric and asymmetric SB models, but it is advised to train separate models for symmetric and asymmetric SB cases with different memory times. The accuracy of the KRR model depends on memory time. By increasing the memory time, the KRR model gets more accurate in the predictions as shown for the hold-out trajectories in Fig. 4. The window-size of the base trajectory $\left(\left|0-t_{m}\right|\right)$ should be wide enough, so that ML model can learn to differentiate among different trajectories. For not wide enough windowsize, KRR model cannot differentiate well between two input trajectories, which results in a rapid deterioration of the accuracy. 

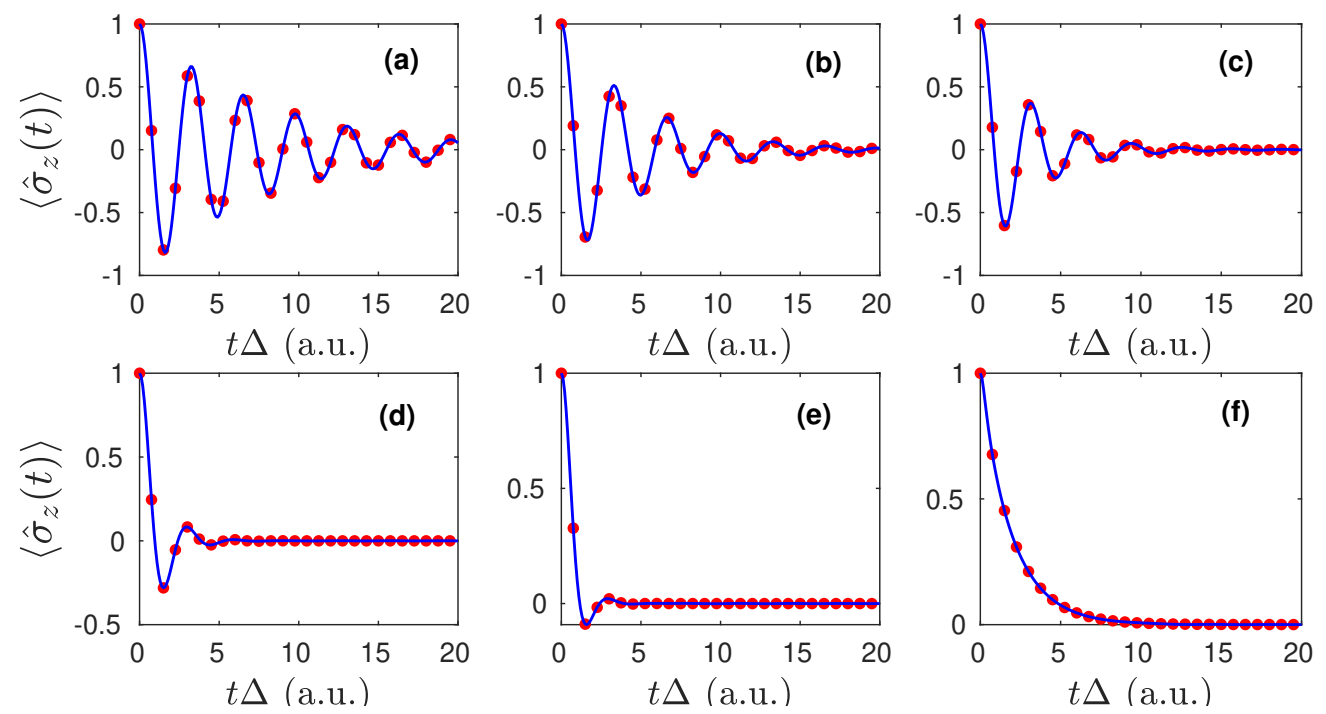

Figure 2: Expectation value of $\hat{\sigma}_{z}$ for symmetric SB model (i.e., $\epsilon=0.0$ ) as a function of time. Results predicted by KRR model (blue line) are compared to the HEOM results (red dots). The adopted parameters are: (a) $\Delta=1.0, \eta=0.2, \omega_{c}=8.0, \beta=1.0$; (b) $\Delta=1.0$, $\eta=0.4, \omega_{c}=10.0, \beta=1.0$; (c) $\Delta=1.0, \eta=0.2, \omega_{c}=10.0, \beta=0.25$; (d) $\Delta=1.0, \eta=0.1$, $\omega_{c}=4.0, \beta=0.1$; (e) $\Delta=1.0, \eta=0.8, \omega_{c}=3.0, \beta=1.0$; (f) $\Delta=1.0, \eta=1.0, \omega_{c}=2.0$, $\beta=0.1$. All parameters are in atomic units.
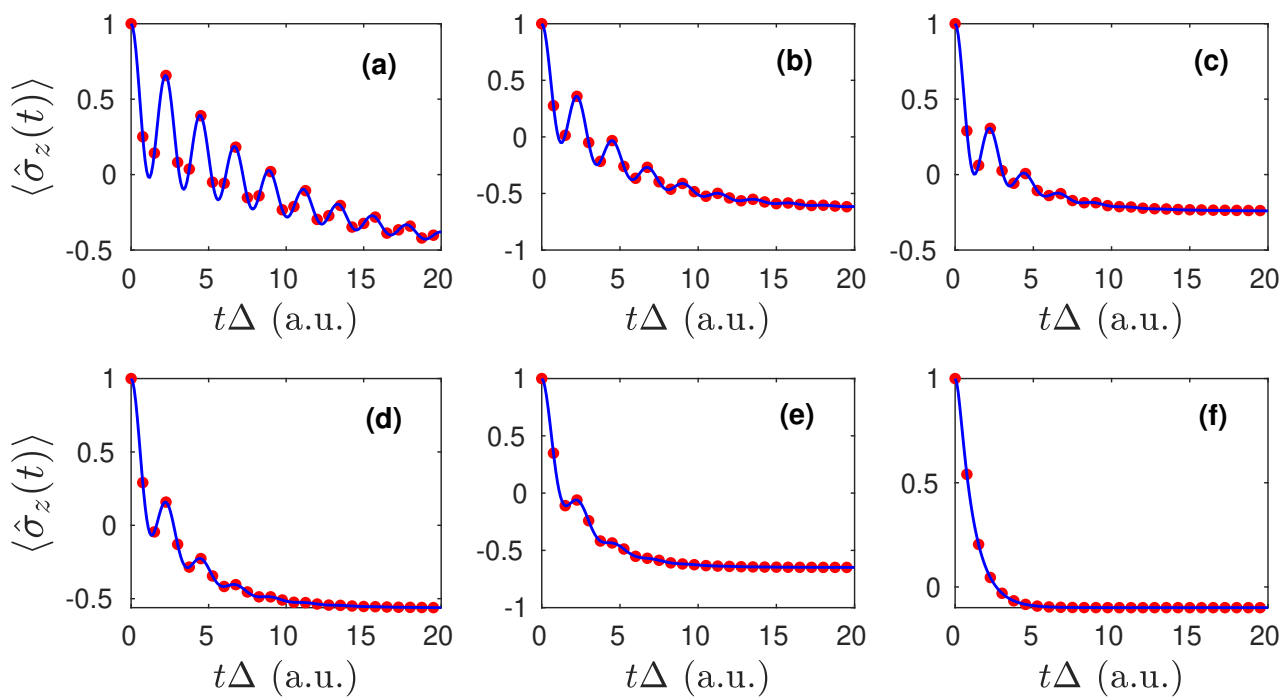

Figure 3: Expectation value of $\hat{\sigma}_{z}$ for asymmetric SB model (i.e., $\epsilon=1.0$ ) as a function of time. Results predicted by KRR model (blue line) are compared to the HEOM results (red dots). The adopted parameters are: (a) $\Delta=1.0, \eta=0.1, \omega_{c}=6.0, \beta=0.75$; (b) $\Delta=1.0$, $\eta=0.3, \omega_{c}=8.0, \beta=1.0$; (c) $\Delta=1.0, \eta=0.2, \omega_{c}=10.0, \beta=0.25 ;$ (d) $\Delta=1.0, \eta=0.4$, $\omega_{c}=8.0, \beta=0.75$; (e) $\Delta=1.0, \eta=0.8, \omega_{c}=10.0, \beta=1.0$; (f) $\Delta=1.0, \eta=0.7, \omega_{c}=10.0$, $\beta=0.1$. All parameters are in atomic units. 


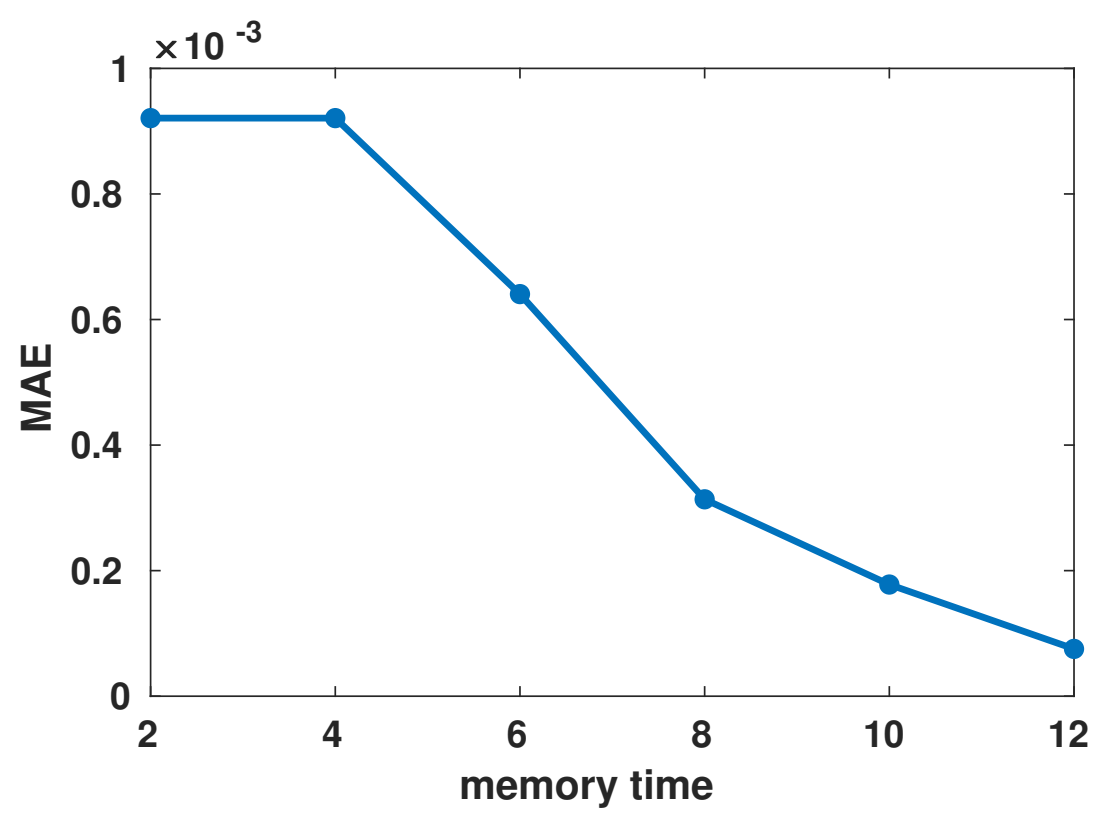

Figure 4: Mean absolute error (MAE) as a function of memory time $t_{m}$. The MAE is calculated for 50 hold-out symmetric trajectories with predictions within the time window $t=12-20$.

As predicted value is included in the input for the next time step, the error accumulation with time is inevitable, which imposes strict requirements on the accuracy of the predictions as for less accurate ML models the quality of trajectories will rapidly deteriorate. Fig. S5 of Supporting Information shows the increase of absolute error in the predictions with the passage of time for the deviating trajectory Fig. S3-(3f) of Supporting Information. As KRR is a nonparametric ML model, the number of parameters increases with the increase of training points, which makes the training very time consuming and requires large memory. To avoid this problem, we should keep the number of points in the training set as minimum as possible. It can be done by using farthest-point sampling, ${ }^{[7]}$ while choosing trajectories for training, or setting $\Delta t_{\text {train }}$ to larger values while ensuring that it will not decrease the accuracy of the KRR predictions too much.

The computational cost-saving by our ML approach is substantial. The amount of time saving depends on the memory time and the trajectory time and in our case it is $75 \%$. This reduction in computational time may allow longer simulations with HEOM model at low 
temperatures, where its cost and memory requirements increase very fast. In contrast, after training, the computational cost of our KRR model remains the same for all cases. On a single Intel(R) Core(TM) i7-10700 CPU @ $2.90 \mathrm{GHz}$, the whole process of prediction and preparing new input at each time-step takes $\approx 2 \mathrm{~min}$ for ML calculations. In the future, our approach can be combined with SEOM, which requires a large number of trajectories to have good convergence in the long-time regime. After generating short time trajectory of timelength $t_{m}$ with SEOM, our approach can be used to predict the long-time dynamics saving a tremendous amount of computational cost, which is currently the topic of our investigations.

\section{Concluding Remarks}

In this article, we have developed an ML approach to study quantum dissipative dynamics. We have demonstrated the ability of kernel ridge regression (KRR) to learn from relatively small amount of training data and predict the long-time quantum dissipative dynamics for two-level SB model without much loss of accuracy. After training, the KRR model requires short-time trajectory as input and as an output, it gives the dynamics for future times. The ability of predictions for future time makes KRR model or in general ML an appealing approach to avoid the calculation of expensive long-time dynamics. It can be combined with other approaches such SEOM, which has bad convergence in long-time regime. Here, we have only demonstrated results for the two-level SB model, but our approach can be extended to multi-level systems given that the data for training is provided. In the end, with the establishment of database with the dynamics data, the ML approach has great potential to become a useful low-cost computational tool for studying the quantum dissipative dynamics.

\section{Acknowledgement}

POD acknowledges funding by the National Natural Science Foundation of China (No. 22003051) and via the Lab project of the State Key Laboratory of Physical Chemistry 
of Solid Surfaces. The authors thank Alexei A. Kananenka for clarifying some issues with his approach.

\section{Supporting Information}

Additional plots for the test trajectories.

\section{Author Contributions}

AU conceived the project, performed all the implementations, simulations, and analysis of the data, as well as written the original version of the manuscript. Both authors carried out the method development, discussed the results, and revised the manuscript. POD acquired funding for the project.

\section{References}

(1) Shor, P. W. Scheme for reducing decoherence in quantum computer memory. Physical review A 1995, 52, R2493.

(2) Pastawski, F.; Clemente, L.; Cirac, J. I. Quantum memories based on engineered dissipation. Physical Review A 2011, 83, 012304.

(3) Wen, X. Quantum Field Theory of Many-Body Systems, Oxford Graduate Texts. 2004.

(4) Le Hur, K.; Henriet, L.; Herviou, L.; Plekhanov, K.; Petrescu, A.; Goren, T.; Schiro, M.; Mora, C.; Orth, P. P. Driven dissipative dynamics and topology of quantum impurity systems. Comptes Rendus Physique 2018, 19, 451-483.

(5) Wurfel, U.; Thorwart, M.; Weber, E. R. Quantum Efficiency in Complex Systems, Part II: From Molecular Aggregates to: Organic Solar Cells; Academic Press, 2011. 
(6) Milošević, M.; Geurts, R. The Ginzburg-Landau theory in application. Physica C: Superconductivity 2010, 470, 791-795.

(7) Huelga, S. F.; Plenio, M. B. Vibrations, quanta and biology. Contemporary Physics 2013, 54, 181-207.

(8) Daley, A. J. Quantum trajectories and open many-body quantum systems. Advances in Physics 2014, 63, 77-149.

(9) Ji, W.; Xu, H.-Q.; Guo, H. Quantum description of transport phenomena: Recent progress. 2014.

(10) Hughes, K. H. Dynamics of open quantum systems; Collaborative Computational Project on Molecular Quantum Dynamics (CCP6), 2006.

(11) Golding, B.; Zimmerman, M. N.; Coppersmith, S. Dissipative quantum tunneling of a single microscopic defect in a mesoscopic metal. Physical review letters 1992, 68, 998.

(12) Vojta, M.; Bulla, R. Kondo effect of impurity moments in d-wave superconductors: Quantum phase transition and spectral properties. Physical Review B 2001, 65, 014511.

(13) Del Valle, E.; Zippilli, S.; Laussy, F. P.; Gonzalez-Tudela, A.; Morigi, G.; Tejedor, C. Two-photon lasing by a single quantum dot in a high-Q microcavity. Physical Review B 2010, 81, 035302 .

(14) Ota, Y.; Iwamoto, S.; Kumagai, N.; Arakawa, Y. Spontaneous two-photon emission from a single quantum dot. Physical review letters 2011, 10\%, 233602.

(15) Reed, M. D.; DiCarlo, L.; Nigg, S. E.; Sun, L.; Frunzio, L.; Girvin, S. M.; Schoelkopf, R. J. Realization of three-qubit quantum error correction with superconducting circuits. Nature 2012, 482, 382-385. 
(16) Georgescu, I. Trapped ion quantum computing turns 25. Nature Reviews Physics 2020, 2, 278-278.

(17) Wilson, K. G. The renormalization group: Critical phenomena and the Kondo problem. Reviews of modern physics 1975, 47, 773.

(18) Bulla, R.; Tong, N.-H.; Vojta, M. Numerical renormalization group for bosonic systems and application to the sub-ohmic spin-boson model. Physical review letters 2003, 91, 170601.

(19) White, S. R. Density matrix formulation for quantum renormalization groups. Physical review letters 1992, 69, 2863.

(20) Wong, H.; Chen, Z.-D. Density matrix renormalization group approach to the spinboson model. Physical Review B 2008, 7r7, 174305.

(21) Egger, R.; Weiss, U. Quantum Monte Carlo simulation of the dynamics of the spinboson model. Zeitschrift für Physik B Condensed Matter 1992, 89, 97-107.

(22) Winter, A.; Rieger, H.; Vojta, M.; Bulla, R. Quantum phase transition in the subohmic spin-boson model: Quantum monte carlo study with a continuous imaginary time cluster algorithm. Physical review letters 2009, 102, 030601.

(23) Chin, A. W.; Rivas, Á.; Huelga, S. F.; Plenio, M. B. Exact mapping between systemreservoir quantum models and semi-infinite discrete chains using orthogonal polynomials. Journal of Mathematical Physics 2010, 51, 092109.

(24) Prior, J.; Chin, A. W.; Huelga, S. F.; Plenio, M. B. Efficient simulation of strong system-environment interactions. Physical review letters 2010, 105, 050404.

(25) Puebla, R.; Zicari, G.; Arrazola, I.; Solano, E.; Paternostro, M.; Casanova, J. Spinboson model as a simulator of non-Markovian multiphoton Jaynes-Cummings models. Symmetry 2019, 11, 695. 
(26) Meyer, H.-D.; Manthe, U.; Cederbaum, L. S. The multi-configurational timedependent Hartree approach. Chemical Physics Letters 1990, 165, 73-78.

(27) Wang, H.; Thoss, M. A multilayer multiconfiguration time-dependent Hartree simulation of the reaction-coordinate spin-boson model employing an interaction picture. The Journal of chemical physics 2017, 146, 124112.

(28) Wang, H.; Thoss, M. Multilayer formulation of the multiconfiguration time-dependent Hartree theory. The Journal of chemical physics 2003, 119, 1289-1299.

(29) Nakajima, S. On quantum theory of transport phenomena: steady diffusion. Progress of Theoretical Physics 1958, 20, 948-959.

(30) Zwanzig, R. Ensemble method in the theory of irreversibility. The Journal of Chemical Physics 1960, 33, 1338-1341.

(31) Cerrillo, J.; Cao, J. Non-Markovian dynamical maps: numerical processing of open quantum trajectories. Physical review letters 2014, 112, 110401.

(32) Kananenka, A. A.; Hsieh, C.-Y.; Cao, J.; Geva, E. Accurate Long-Time Mixed Quantum-Classical Liouville Dynamics via the Transfer Tensor Method. The journal of physical chemistry letters 2016, 7, 4809-4814.

(33) Buser, M.; Cerrillo, J.; Schaller, G.; Cao, J. Initial system-environment correlations via the transfer-tensor method. Physical Review A 2017, 96, 062122.

(34) Zhou, N.; Zhang, Y.; Lü, Z.; Zhao, Y. Variational Study of the Two-Impurity SpinBoson Model with a Common Ohmic Bath: Ground-State Phase Transitions. Annalen der Physik 2018, 530, 1800120.

(35) Miller, W. H.; Cotton, S. J. Classical molecular dynamics simulation of electronically non-adiabatic processes. Faraday discussions 2017, 195, 9-30. 
(36) Cotton, S. J.; Miller, W. H. The symmetrical quasi-classical model for electronically non-adiabatic processes applied to energy transfer dynamics in site-exciton models of light-harvesting complexes. Journal of chemical theory and computation 2016, 12, 983-991.

(37) Cotton, S. J.; Miller, W. H. A symmetrical quasi-classical windowing model for the molecular dynamics treatment of non-adiabatic processes involving many electronic states. The Journal of chemical physics 2019, 150, 104101.

(38) Meyer, H.-D.; Miller, W. H. Classical models for electronic degrees of freedom: Derivation via spin analogy and application to $\mathrm{F}^{*}+\mathrm{H} 2 \rightarrow \mathrm{F}+\mathrm{H} 2$. The Journal of Chemical Physics 1979, 71, 2156-2169.

(39) Stock, G.; Thoss, M. Semiclassical description of nonadiabatic quantum dynamics. Physical review letters 1997, 78, 578.

(40) Runeson, J. E.; Richardson, J. O. Generalized spin mapping for quantum-classical dynamics. The Journal of chemical physics 2020, 152, 084110.

(41) Mannouch, J. R.; Richardson, J. O. A partially linearized spin-mapping approach for nonadiabatic dynamics. I. Derivation of the theory. The Journal of Chemical Physics 2020, 153, 194109.

(42) Makarov, D. E.; Makri, N. Path integrals for dissipative systems by tensor multiplication. Condensed phase quantum dynamics for arbitrarily long time. Chemical physics letters 1994, 221, 482-491.

(43) Makri, N. Numerical path integral techniques for long time dynamics of quantum dissipative systems. Journal of Mathematical Physics 1995, 36, 2430-2457.

(44) Yan, Y.-a.; Yang, F.; Liu, Y.; Shao, J. Hierarchical approach based on stochastic decoupling to dissipative systems. Chemical physics letters 2004, 395, 216-221. 
(45) Tanimura, Y. Stochastic Liouville, Langevin, Fokker-Planck, and master equation approaches to quantum dissipative systems. Journal of the Physical Society of Japan 2006, 75, 082001.

(46) Jin, J.; Zheng, X.; Yan, Y. Exact dynamics of dissipative electronic systems and quantum transport: Hierarchical equations of motion approach. The Journal of chemical physics 2008, 128, 234703.

(47) Shi, Q.; Chen, L.; Nan, G.; Xu, R.-X.; Yan, Y. Efficient hierarchical Liouville space propagator to quantum dissipative dynamics. The Journal of chemical physics 2009, 130,084105 .

(48) Hu, J.; Xu, R.-X.; Yan, Y. Communication: Padé spectrum decomposition of Fermi function and Bose function. 2010.

(49) Liu, H.; Zhu, L.; Bai, S.; Shi, Q. Reduced quantum dynamics with arbitrary bath spectral densities: Hierarchical equations of motion based on several different bath decomposition schemes. The Journal of chemical physics 2014, 140, 134106.

(50) Gong, H.; Ullah, A.; Ye, L.; Zheng, X.; Yan, Y. Quantum entanglement of parallelcoupled double quantum dots: A theoretical study using the hierarchical equations of motion approach. Chinese Journal of Chemical Physics 2018, 31, 510.

(51) Han, L.; Zhang, H.-D.; Zheng, X.; Yan, Y. On the exact truncation tier of fermionic hierarchical equations of motion. The Journal of chemical physics 2018, 148, 234108.

(52) Cui, L.; Zhang, H.-D.; Zheng, X.; Xu, R.-X.; Yan, Y. Highly efficient and accurate sum-over-poles expansion of Fermi and Bose functions at near zero temperatures: Fano spectrum decomposition scheme. The Journal of chemical physics 2019, 151, 024110.

(53) Zhang, H.-D.; Cui, L.; Gong, H.; Xu, R.-X.; Zheng, X.; Yan, Y. Hierarchical equa- 
tions of motion method based on Fano spectrum decomposition for low temperature environments. The Journal of chemical physics 2020, 152, 064107.

(54) Tanimura, Y.; Kubo, R. Time evolution of a quantum system in contact with a nearly Gaussian-Markoffian noise bath. Journal of the Physical Society of Japan 1989, 58, $101-114$.

(55) Stockburger, J. T.; Mak, C. Dynamical simulation of current fluctuations in a dissipative two-state system. Physical review letters 1998, 80, 2657.

(56) Stockburger, J. T.; Grabert, H. Non-Markovian quantum state diffusion. Chemical Physics 2001, 268, 249-256.

(57) Stockburger, J. T.; Grabert, H. Exact c-number representation of non-Markovian quantum dissipation. Physical review letters 2002, 88, 170407.

(58) Stockburger, J. T. Simulating spin-boson dynamics with stochastic liouville--von neumann equations. Chemical physics 2004, 296, 159-169.

(59) Koch, W.; Großmann, F.; Stockburger, J. T.; Ankerhold, J. Non-Markovian dissipative semiclassical dynamics. Physical review letters 2008, 100, 230402.

(60) Stockburger, J. T. Exact propagation of open quantum systems in a system-reservoir context. EPL (Europhysics Letters) 2016, 115, 40010.

(61) Schmitz, K.; Stockburger, J. T. A variance reduction technique for the stochastic Liouville--von Neumann equation. The European Physical Journal Special Topics 2019, 227, 1929-1937.

(62) Shao, J. Decoupling quantum dissipation interaction via stochastic fields. The Journal of chemical physics 2004, 120, 5053-5056.

(63) Zhou, Y.; Yan, Y.; Shao, J. Stochastic simulation of quantum dissipative dynamics. EPL (Europhysics Letters) 2005, 72, 334. 
(64) Shao, J. Rigorous representation and exact simulation of real Gaussian stationary processes. Chemical Physics 2010, 375, 378-379.

(65) Hsieh, C.-Y.; Cao, J. A unified stochastic formulation of dissipative quantum dynamics. II. Beyond linear response of spin baths. The Journal of chemical physics 2018, $148,014104$.

(66) McCaul, G.; Lorenz, C.; Kantorovich, L. Partition-free approach to open quantum systems in harmonic environments: An exact stochastic Liouville equation. Physical Review B 2017, 95, 125124.

(67) Ke, Y.; Zhao, Y. Hierarchy of forward-backward stochastic Schrödinger equation. The Journal of chemical physics 2016, 145, 024101.

(68) Ke, Y.; Zhao, Y. An extension of stochastic hierarchy equations of motion for the equilibrium correlation functions. The Journal of chemical physics 2017, 146, 214105.

(69) Han, L.; Ullah, A.; Yan, Y.-A.; Zheng, X.; Yan, Y.; Chernyak, V. Stochastic equation of motion approach to fermionic dissipative dynamics. I. Formalism. The Journal of Chemical Physics 2020, 152, 204105.

(70) Ullah, A.; Han, L.; Yan, Y.-A.; Zheng, X.; Yan, Y.; Chernyak, V. Stochastic equation of motion approach to fermionic dissipative dynamics. II. Numerical implementation. The Journal of Chemical Physics 2020, 152, 204106.

(71) Han, L.; Chernyak, V.; Yan, Y.-A.; Zheng, X.; Yan, Y. Stochastic Representation of Non-Markovian Fermionic Quantum Dissipation. Physical review letters 2019, 123, 050601.

(72) Zhong, X.; Zhao, Y. Non-Markovian stochastic Schrödinger equation at finite temperatures for charge carrier dynamics in organic crystals. The Journal of chemical physics 2013, 138, 014111. 
(73) Wang, Y.-C.; Ke, Y.; Zhao, Y. The hierarchical and perturbative forms of stochastic Schrödinger equations and their applications to carrier dynamics in organic materials. Wiley Interdisciplinary Reviews: Computational Molecular Science 2019, 9, e1375.

(74) Lian, M.; Wang, Y.-C.; Ke, Y.; Zhao, Y. Non-Markovian stochastic Schrödinger equation in k-space toward the calculation of carrier dynamics in organic semiconductors. The Journal of chemical physics 2019, 151, 044115.

(75) Wang, Y.-C.; Zhao, Y. The hierarchical stochastic Schrödinger equations: Theory and applications. Chinese Journal of Chemical Physics 2020, 33, 653-667.

(76) Dral, P. O. Quantum chemistry assisted by machine learning. Advances in Quantum Chemistry 2020, 81, 291-324.

(77) von Lilienfeld, O. A.; Müller, K.-R.; Tkatchenko, A. Exploring chemical compound space with quantum-based machine learning. Nat. Rev. Chem. 2020, 4, 347-358.

(78) Unke, O. T.; Chmiela, S.; Sauceda, H. E.; Gastegger, M.; Poltavsky, I.; Schutt, K. T.; Tkatchenko, A.; Muller, K. R. Machine Learning Force Fields. Chem. Rev. 2021, DOI: 10.1021/acs.chemrev.0c01111.

(79) Manzhos, S.; Carrington, J., T. Neural Network Potential Energy Surfaces for Small Molecules and Reactions. Chem. Rev. 2020, DOI: 10.1021/acs.chemrev.0c00665.

(80) Wang, J.; Chmiela, S.; Müller, K.-R.; Noé, F.; Clementi, C. Ensemble learning of coarse-grained molecular dynamics force fields with a kernel approach. The Journal of Chemical Physics 2020, 152, 194106.

(81) Veit, M.; Wilkins, D. M.; Yang, Y.; DiStasio Jr, R. A.; Ceriotti, M. Predicting molecular dipole moments by combining atomic partial charges and atomic dipoles. The Journal of Chemical Physics 2020, 153, 024113. 
(82) Morita, K.; Davies, D. W.; Butler, K. T.; Walsh, A. Modeling the dielectric constants of crystals using machine learning. The Journal of Chemical Physics 2020, 153, 024503.

(83) Xue, B.-X.; Barbatti, M.; Dral, P. O. Machine Learning for Absorption Cross Sections. The Journal of Physical Chemistry A 2020, 124, 7199-7210.

(84) Dral, P. O.; Barbatti, M. Molecular excited states through a machine learning lens. Nature Reviews Chemistry 2021, 1-18.

(85) Westermayr, J.; Marquetand, P. Machine learning and excited-state molecular dynamics. Mach. Learn.: Sci. Technol. 2020, 1, 043001.

(86) Ueno, S.; Tanimura, Y. Modeling and simulating the excited-state dynamics of a system with condensed phases: A machine learning approach. Journal of Chemical Theory and Computation 2021,

(87) Yang, B.; He, B.; Wan, J.; Kubal, S.; Zhao, Y. Applications of neural networks to dynamics simulation of Landau-Zener transitions. Chemical Physics 2020, 528, 110509.

(88) Bandyopadhyay, S.; Huang, Z.; Sun, K.; Zhao, Y. Applications of neural networks to the simulation of dynamics of open quantum systems. Chemical Physics 2018, 515, $272-278$.

(89) Herrera Rodríguez, L. E.; Kananenka, A. A. Convolutional Neural Networks for Long Time Dissipative Quantum Dynamics. The Journal of Physical Chemistry Letters 2021, 12, 2476-2483.

(90) Kamath, A.; Vargas-Hernández, R. A.; Krems, R. V.; Carrington, J., T.; Manzhos, S. Neural networks vs Gaussian process regression for representing potential energy surfaces: A comparative study of fit quality and vibrational spectrum accuracy. J. Chem. Phys. 2018, 148, 241702. 
(91) Nguyen, T. T.; Szekely, E.; Imbalzano, G.; Behler, J.; Csányi, G.; Ceriotti, M.; Gotz, A. W.; Paesani, F. Comparison of permutationally invariant polynomials, neural networks, and Gaussian approximation potentials in representing water interactions through many-body expansions. J. Chem. Phys. 2018, 148, 241725.

(92) Hansen, K.; Montavon, G.; Biegler, F.; Fazli, S.; Rupp, M.; Scheffler, M.; von Lilienfeld, O. A.; Tkatchenko, A.; Müller, K.-R. Assessment and Validation of Machine Learning Methods for Predicting Molecular Atomization Energies. J. Chem. Theory Comput. 2013, 9, 3404-3419.

(93) Stulp, F.; Sigaud, O. Many regression algorithms, one unified model: A review. Neural Networks 2015, 69, 60-79.

(94) Hastie, T. Tibshirani, r. and Friedman, J.(2009): The elements of statistical learning. Data mining, inference, and prediction. 2008.

(95) Rupp, M. Machine learning for quantum mechanics in a nutshell. International Journal of Quantum Chemistry 2015, 115, 1058-1073.

(96) Dral, P. O. MLatom: A program package for quantum chemical research assisted by machine learning. Journal of computational chemistry 2019, 40, 2339-2347.

(97) Dral, P. O.; Ge, F.; Xue, B.-X.; Hou, Y.-F.; Pinheiro, M.; Huang, J.; Barbatti, M. MLatom 2: An Integrative Platform for Atomistic Machine Learning. Topics in Current Chemistry 2021, 379, 1-41.

(98) Dral, P. O.; Xue, B.-X.; Ge, F.; Hou, Y.-F.; Pinheiro Jr, M. MLatom: a Package for Atomistic Simulations with Machine Learning. http://MLatom.com (accessed on August 9, 2021). Xiamen University: Xiamen, China, 2013-2021.

(99) Witten, I. H.; Frank, E.; Hall, M. A.; Pal, C.; DATA, M. Practical machine learning tools and techniques. DATA MINING. 2005; p 4. 
(100) Johansson, J. R.; Nation, P. D.; Nori, F. QuTiP: An open-source Python framework for the dynamics of open quantum systems. Computer Physics Communications 2012, $183,1760-1772$.

(101) Häse, F.; Kreisbeck, C.; Aspuru-Guzik, A. Machine learning for quantum dynamics: deep learning of excitation energy transfer properties. Chem. Sci. 2017, 8, 8419-8426. 
Supporting information for:

\title{
Speeding up quantum dissipative dynamics of open systems with kernel methods
}

\author{
Arif Ullah* and Pavlo O. Dral* \\ State Key Laboratory of Physical Chemistry of Solid Surfaces, Fujian Provincial Key \\ Laboratory of Theoretical and Computational Chemistry, Department of Chemistry, and \\ College of Chemistry and Chemical Engineering, Xiamen University, Xiamen 361005,
} China

E-mail: ua2024@xmu.edu.cn dral@xmu.edu.cn

Table S1: Parameters for the sub-plots shown in Fig. S1 S4. The common parameter is $\Delta=1.0$. All parameters are in atomic units (a.u.).

\begin{tabular}{|l|l|l|l|l|l|l|l|l|l|}
\hline sub-plots & $\epsilon$ & $\lambda$ & $\omega_{c}$ & $\beta$ & sub-plots & $\epsilon$ & $\lambda$ & $\omega_{c}$ & $\beta$ \\
\hline$(1 \mathrm{a})$ & 0.0 & 0.1 & 1.0 & 0.5 & $(3 \mathrm{a})$ & 1.0 & 0.1 & 2.0 & 1.0 \\
$(1 \mathrm{~b})$ & 0.0 & 0.1 & 1.0 & 0.25 & $(3 \mathrm{~b})$ & 1.0 & 0.1 & 3.0 & 0.75 \\
$(1 \mathrm{c})$ & 0.0 & 0.1 & 4.0 & 0.75 & $(3 \mathrm{c})$ & 1.0 & 0.1 & 7.0 & 0.5 \\
$(1 \mathrm{~d})$ & 0.0 & 0.1 & 6.0 & 0.5 & $(3 \mathrm{~d})$ & 1.0 & 0.1 & 10.0 & 0.1 \\
$(1 \mathrm{e})$ & 0.0 & 0.2 & 1.0 & 0.75 & $(3 \mathrm{e})$ & 1.0 & 0.2 & 1.0 & 0.25 \\
$(1 \mathrm{f})$ & 0.0 & 0.2 & 3.0 & 0.5 & $(3 \mathrm{f})$ & 1.0 & 0.2 & 1.0 & 1.0 \\
$(1 \mathrm{~g})$ & 0.0 & 0.2 & 7.0 & 1.0 & $(3 \mathrm{~g})$ & 1.0 & 0.2 & 2.0 & 0.75 \\
$(1 \mathrm{~h})$ & 0.0 & 0.3 & 1.0 & 0.25 & $(3 \mathrm{~h})$ & 1.0 & 0.2 & 4.0 & 0.1 \\
\hline
\end{tabular}

Continued on next page 
Table S1 - Continued from previous page

\begin{tabular}{|c|c|c|c|c|c|c|c|c|c|}
\hline sub-plots & $\epsilon$ & $\lambda$ & $\omega_{c}$ & $\beta$ & sub-plots & $\epsilon$ & $\lambda$ & $\omega_{c}$ & $\beta$ \\
\hline (1i) & 0.0 & 0.3 & 2.0 & 1.0 & $(3 \mathrm{i})$ & 1.0 & 0.2 & 4.0 & 0.5 \\
\hline$(1 \mathrm{j})$ & 0.0 & 0.3 & 8.0 & 0.5 & $(3 \mathrm{j})$ & 1.0 & 0.2 & 5.0 & 0.75 \\
\hline$(1 \mathrm{k})$ & 0.0 & 0.3 & 8.0 & 0.25 & $(3 \mathrm{k})$ & 1.0 & 0.2 & 6.0 & 1.0 \\
\hline (11) & 0.0 & 0.3 & 8.0 & 0.75 & $(3 \mathrm{l})$ & 1.0 & 0.2 & 7.0 & 0.25 \\
\hline$(1 \mathrm{~m})$ & 0.0 & 0.4 & 2.0 & 0.1 & $(3 \mathrm{~m})$ & 1.0 & 0.2 & 8.0 & 0.5 \\
\hline$(1 \mathrm{n})$ & 0.0 & 0.4 & 2.0 & 0.25 & $(3 n)$ & 1.0 & 0.2 & 8.0 & 0.25 \\
\hline (10) & 0.0 & 0.4 & 3.0 & 0.75 & (3o) & 1.0 & 0.3 & 2.0 & 0.25 \\
\hline (1p) & 0.0 & 0.4 & 6.0 & 0.1 & $(3 p)$ & 1.0 & 0.3 & 4.0 & 1.0 \\
\hline (1q) & 0.0 & 0.4 & 6.0 & 0.75 & $(3 q)$ & 1.0 & 0.3 & 7.0 & 0.25 \\
\hline$(1 \mathrm{r})$ & 0.0 & 0.4 & 7.0 & 0.25 & $(3 r)$ & 1.0 & 0.3 & 8.0 & 0.75 \\
\hline (1s) & 0.0 & 0.4 & 8.0 & 1.0 & $(3 \mathrm{~s})$ & 1.0 & 0.3 & 10.0 & 1.0 \\
\hline$(1 \mathrm{t})$ & 0.0 & 0.5 & 1.0 & 0.5 & $(3 \mathrm{t})$ & 1.0 & 0.4 & 2.0 & 0.75 \\
\hline$(1 u)$ & 0.0 & 0.5 & 1.0 & 0.25 & $(3 \mathrm{u})$ & 1.0 & 0.4 & 7.0 & 0.1 \\
\hline$(1 \mathrm{v})$ & 0.0 & 0.5 & 1.0 & 1.0 & $(3 \mathrm{v})$ & 1.0 & 0.4 & 7.0 & 1.0 \\
\hline$(1 \mathrm{w})$ & 0.0 & 0.5 & 6.0 & 0.5 & $(3 \mathrm{w})$ & 1.0 & 0.5 & 1.0 & 0.5 \\
\hline$(1 \mathrm{x})$ & 0.0 & 0.5 & 8.0 & 0.1 & $(3 \mathrm{x})$ & 1.0 & 0.5 & 3.0 & 0.5 \\
\hline$(2 a)$ & 0.0 & 0.5 & 9.0 & 0.75 & $(4 a)$ & 1.0 & 0.5 & 8.0 & 0.25 \\
\hline$(2 b)$ & 0.0 & 0.6 & 2.0 & 0.25 & $(4 b)$ & 1.0 & 0.5 & 8.0 & 0.75 \\
\hline$(2 \mathrm{c})$ & 0.0 & 0.6 & 2.0 & 1.0 & $(4 c)$ & 1.0 & 0.6 & 1.0 & 0.1 \\
\hline$(2 \mathrm{~d})$ & 0.0 & 0.6 & 6.0 & 0.5 & $(4 d)$ & 1.0 & 0.6 & 1.0 & 1.0 \\
\hline$(2 \mathrm{e})$ & 0.0 & 0.6 & 10.0 & 0.75 & $(4 \mathrm{e})$ & 1.0 & 0.6 & 4.0 & 1.0 \\
\hline$(2 \mathrm{f})$ & 0.0 & 0.7 & 6.0 & 0.1 & $(4 f)$ & 1.0 & 0.7 & 1.0 & 0.75 \\
\hline$(2 \mathrm{~g})$ & 0.0 & 0.7 & 6.0 & 0.75 & $(4 \mathrm{~g})$ & 1.0 & 0.7 & 5.0 & 0.25 \\
\hline$(2 \mathrm{~h})$ & 0.0 & 0.7 & 7.0 & 0.5 & $(4 \mathrm{~h})$ & 1.0 & 0.7 & 6.0 & 0.5 \\
\hline
\end{tabular}


Table S1 - Continued from previous page

\begin{tabular}{|l|l|l|l|l|l|l|l|l|l|}
\hline sub-plots & $\epsilon$ & $\lambda$ & $\omega_{c}$ & $\beta$ & sub-plots & $\epsilon$ & $\lambda$ & $\omega_{c}$ & $\beta$ \\
\hline$(2 \mathrm{i})$ & 0.0 & 0.7 & 7.0 & 0.25 & $(4 \mathrm{i})$ & 1.0 & 0.7 & 9.0 & 0.5 \\
$(2 \mathrm{j})$ & 0.0 & 0.7 & 8.0 & 0.1 & $(4 \mathrm{j})$ & 1.0 & 0.8 & 2.0 & 0.1 \\
$(2 \mathrm{k})$ & 0.0 & 0.7 & 8.0 & 0.75 & $(4 \mathrm{k})$ & 1.0 & 0.8 & 2.0 & 0.25 \\
$(2 \mathrm{l})$ & 0.0 & 0.7 & 9.0 & 0.1 & $(4 \mathrm{l})$ & 1.0 & 0.8 & 6.0 & 0.1 \\
$(2 \mathrm{~m})$ & 0.0 & 0.9 & 4.0 & 0.5 & $(4 \mathrm{~m})$ & 1.0 & 0.8 & 10.0 & 0.75 \\
$(2 \mathrm{n})$ & 0.0 & 0.9 & 10.0 & 1.0 & $(4 \mathrm{n})$ & 1.0 & 0.6 & 4.0 & 0.1 \\
$(2 \mathrm{o})$ & 0.0 & 1.0 & 5.0 & 0.75 & $(4 \mathrm{o})$ & 1.0 & 0.6 & 4.0 & 0.25 \\
$(2 \mathrm{p})$ & 0.0 & 1.0 & 6.0 & 0.25 & $(4 \mathrm{p})$ & 1.0 & 0.6 & 6.0 & 0.5 \\
$(2 \mathrm{q})$ & 0.0 & 1.0 & 8.0 & 0.5 & $(4 \mathrm{q})$ & 1.0 & 0.6 & 7.0 & 0.1 \\
$(2 \mathrm{r})$ & 0.0 & 1.0 & 8.0 & 1.0 & $(4 \mathrm{r})$ & 1.0 & 0.6 & 7.0 & 0.5 \\
$(2 \mathrm{~s})$ & 0.0 & 1.0 & 9.0 & 0.1 & $(4 \mathrm{~s})$ & 1.0 & 0.6 & 10.0 & 0.1 \\
$(2 \mathrm{t})$ & 0.0 & 1.0 & 10.0 & 0.1 & $(4 \mathrm{t})$ & 1.0 & 1.0 & 3.0 & 0.5 \\
\hline
\end{tabular}



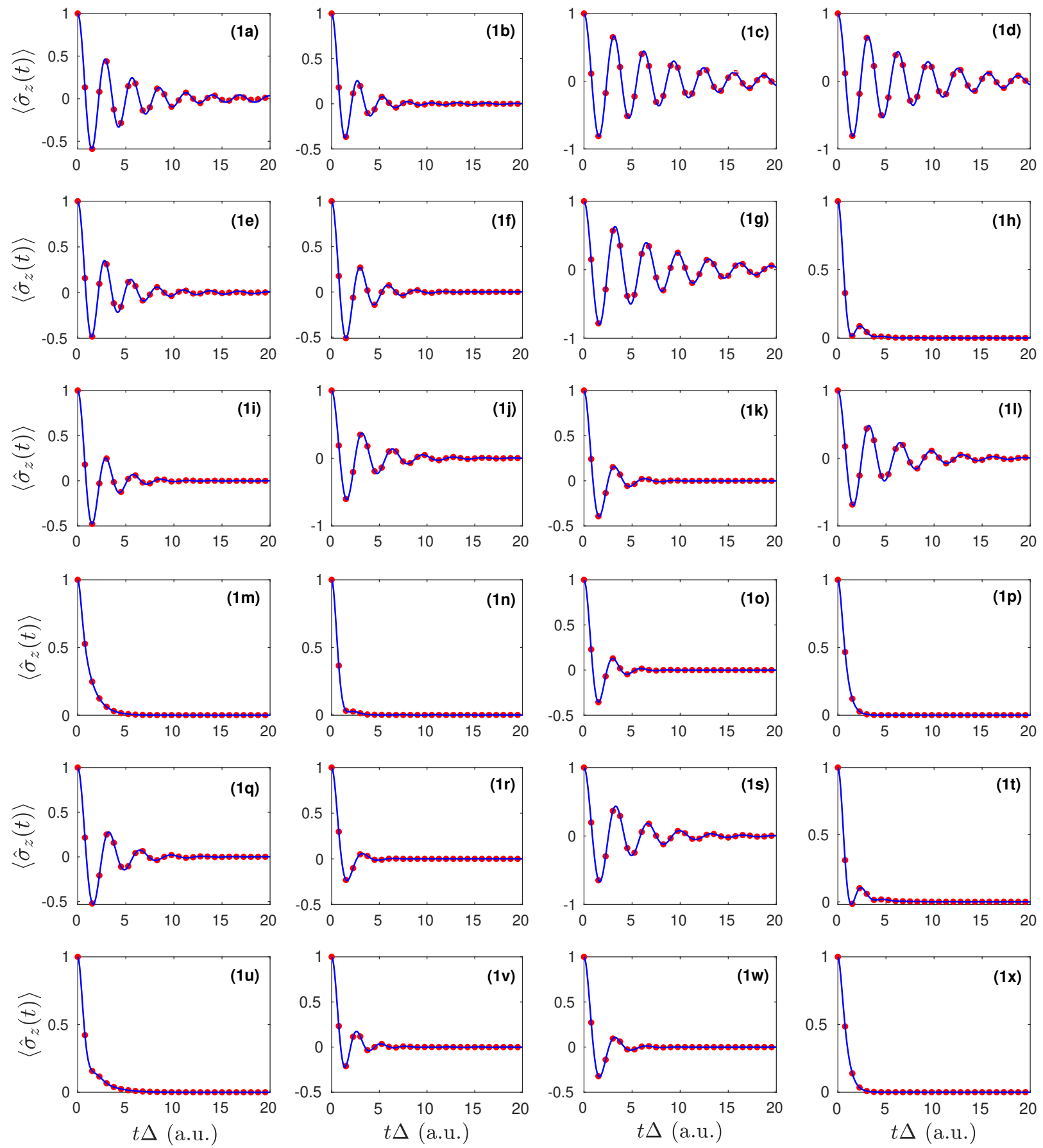

Figure S1: Expectation value of $\hat{\sigma}_{z}$ for symmetric SB model as a function of time. Results predicted by KRR model (blue line) are compared to the HEOM results (red dots). The adopted parameters are given in Table S1. 

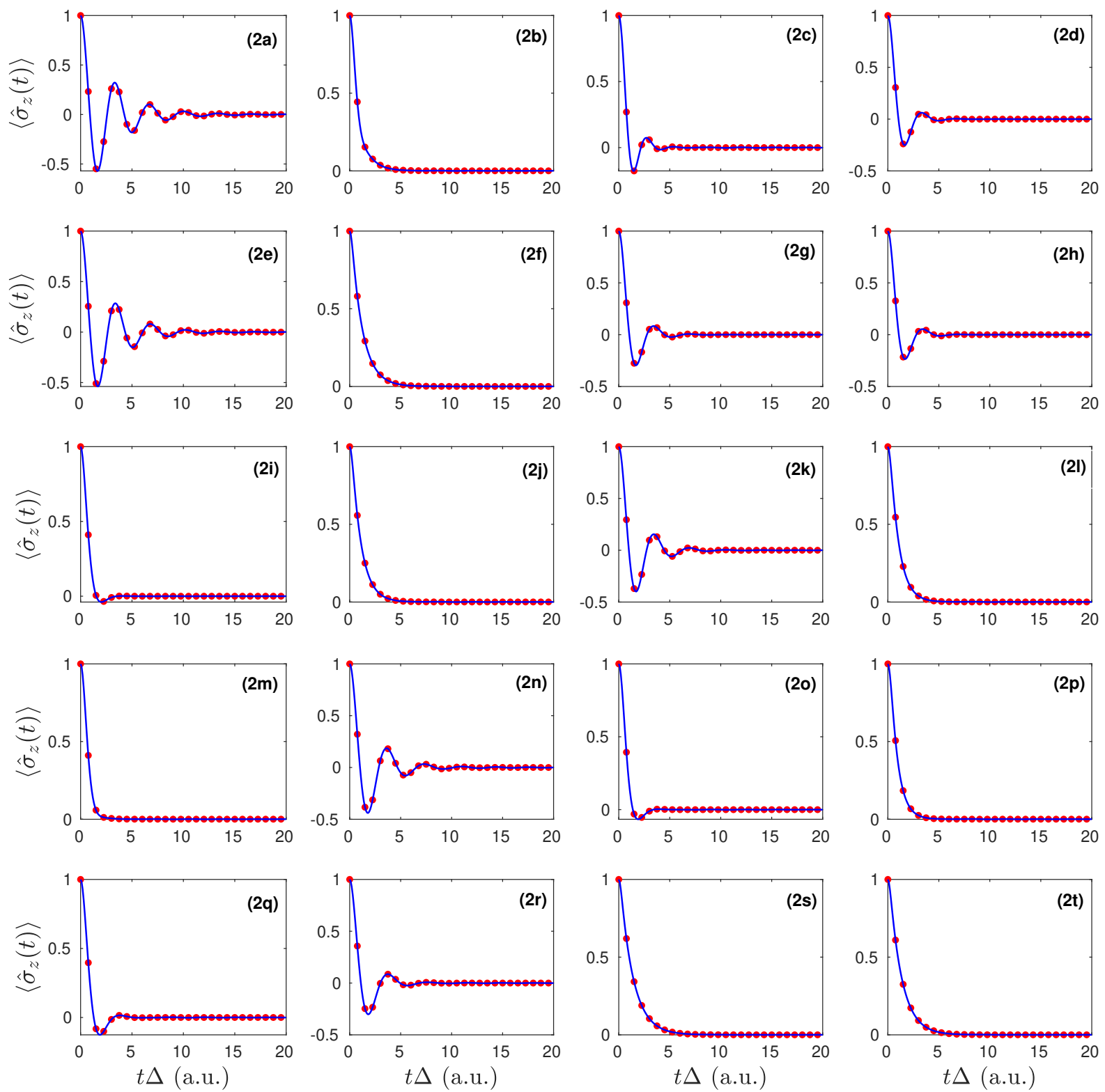

Figure S2: Expectation value of $\hat{\sigma}_{z}$ for symmetric SB model as a function of time. Results predicted by KRR model (blue line) are compared to the HEOM results (red dots). The adopted parameters are given in Table S1. 

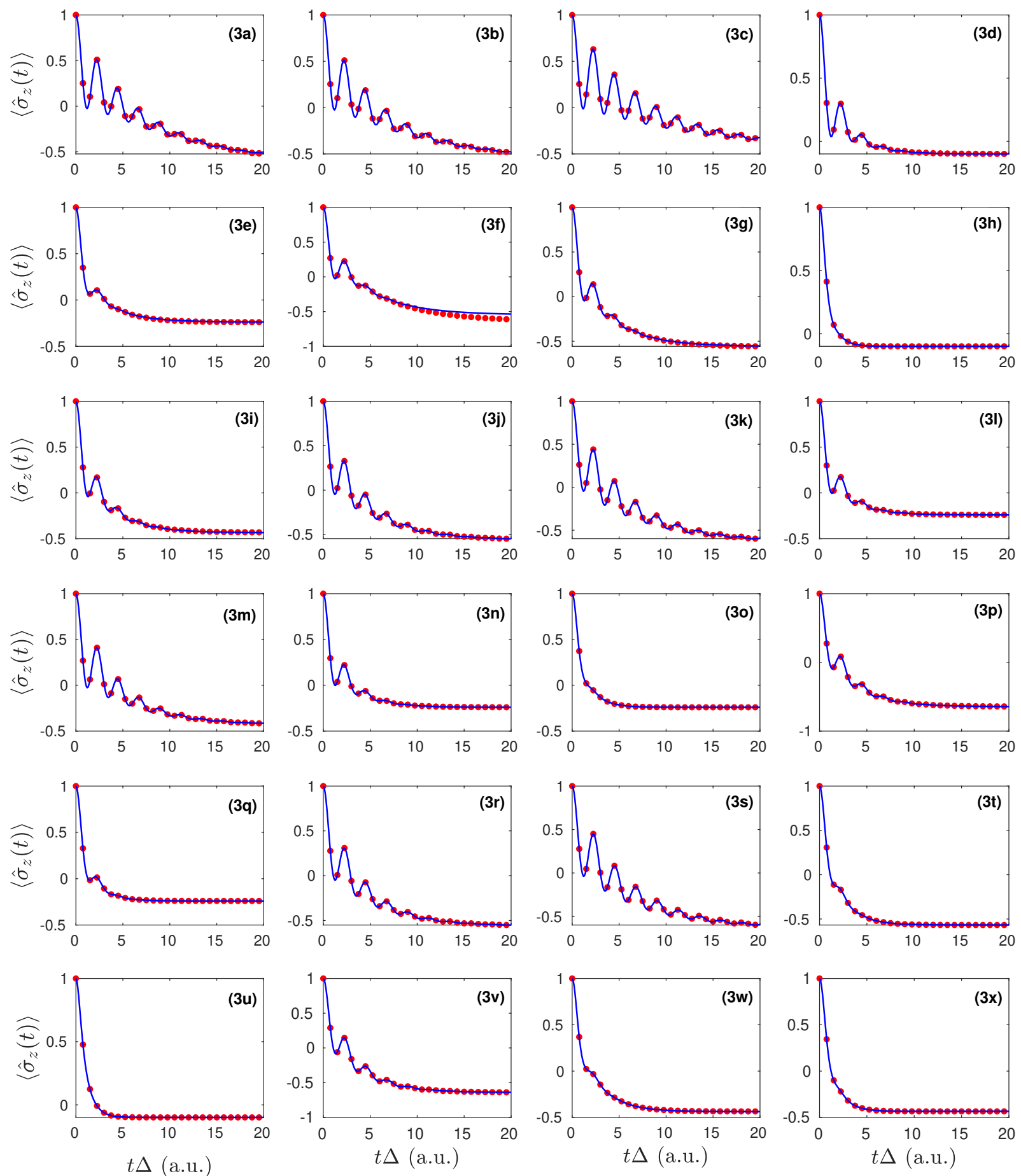

Figure S3: Expectation value of $\hat{\sigma}_{z}$ for asymmetric SB model as a function of time. Results predicted by KRR model (blue line) are compared to the HEOM results (red dots). The adopted parameters are given in Table S1. 

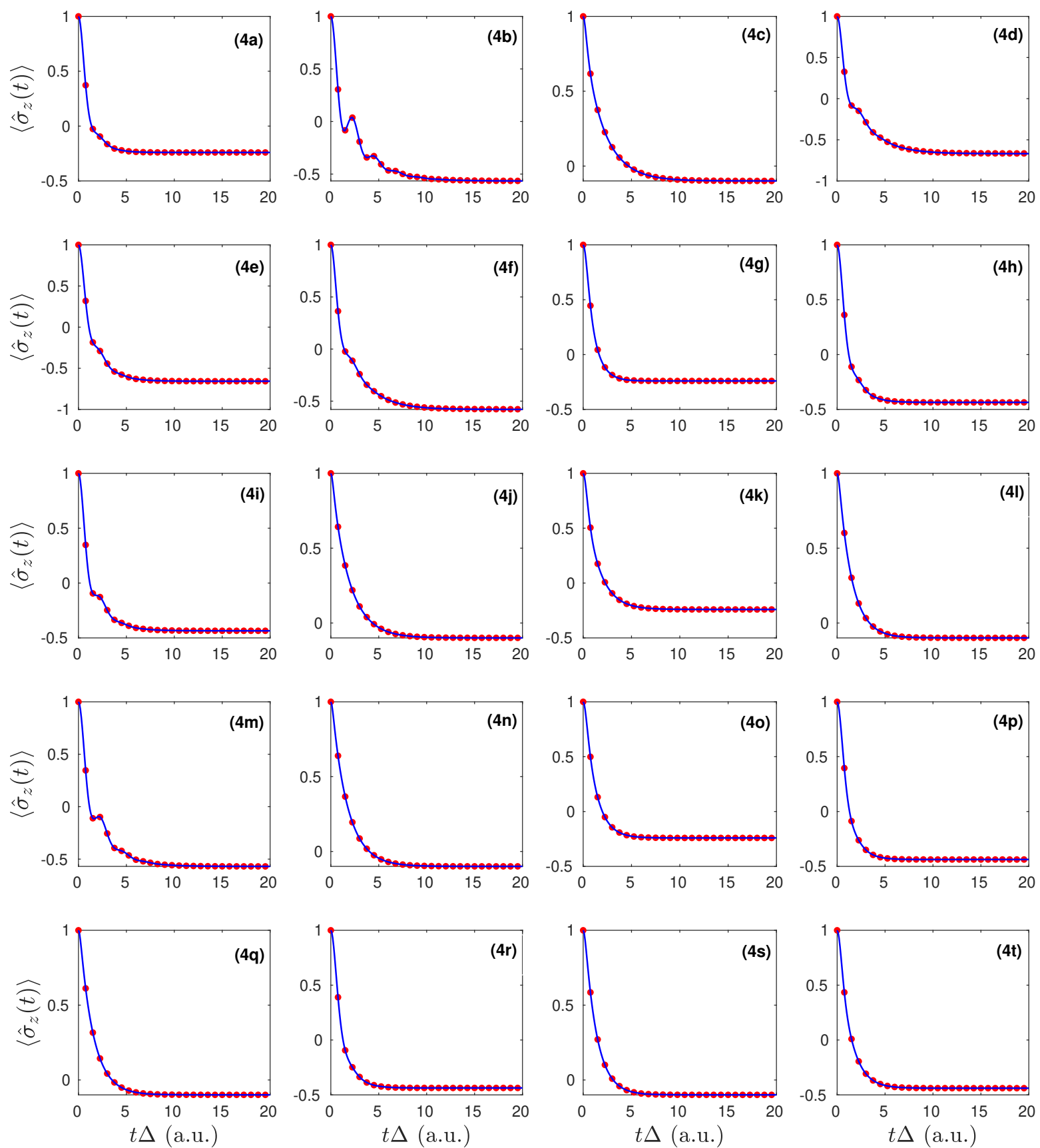

Figure S4: Expectation value of $\hat{\sigma}_{z}$ for asymmetric SB model as a function of time. Results predicted by KRR model (blue line) are compared to the HEOM results (red dots). The adopted parameters are given in Table S1. 


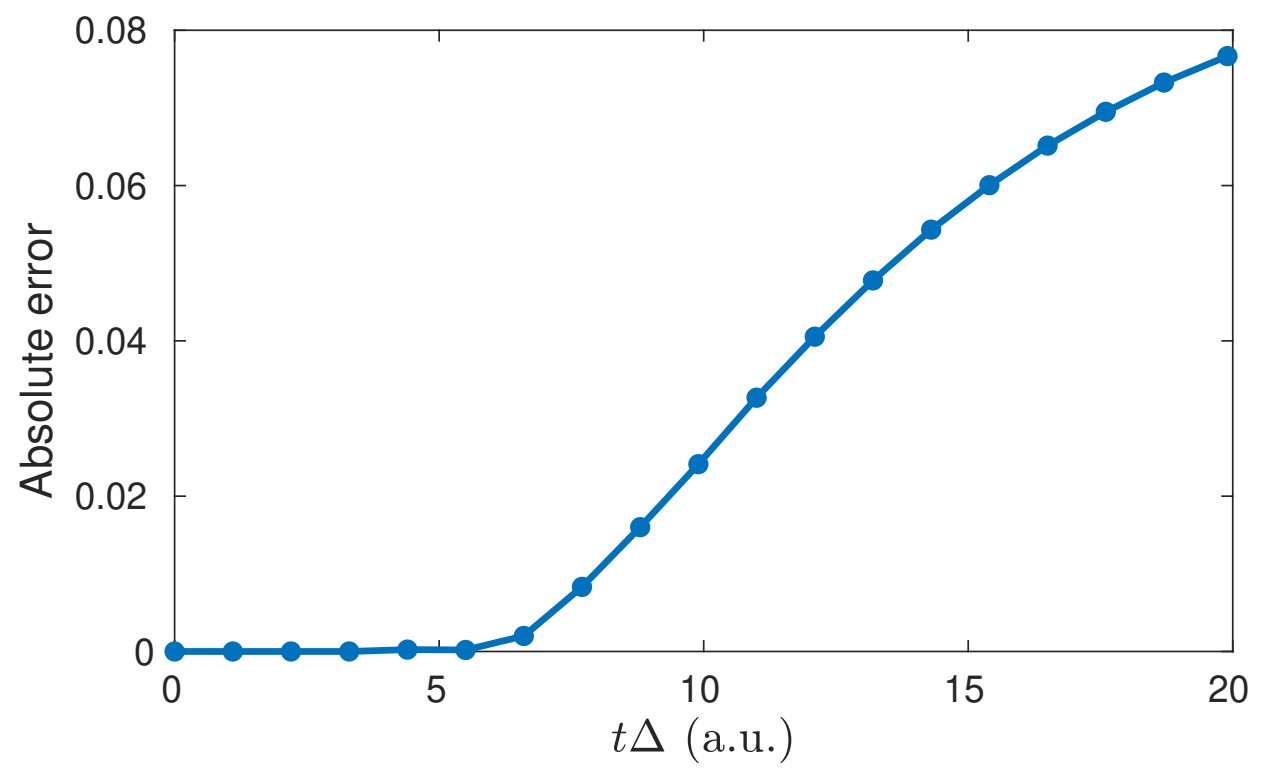

Figure S5: Absolute error $\left|\left\langle\hat{\sigma}_{z}(t)\right\rangle^{\mathrm{HEOM}}-\left\langle\hat{\sigma}_{z}(t)\right\rangle^{\mathrm{KRR}}\right|$ for Fig. $\mathrm{S3}$-(3f) as a function of time. 